\title{
Fully Symmetrized VB Based Technique for Solving Exchange Hamiltonians of Molecular Magnets
}

\author{
$\operatorname{Shaon~Sahoo~}^{a, 1}, \operatorname{Raghunathan}_{\operatorname{Rajamani}^{b}, 2}$, S. Ramasesha ${ }^{b, 3}$ and Diptiman $\operatorname{Sen}^{c, 4}$
}

a. Department of Physics, Indian Institute of Science, Bangalore 560012, India.

b. Solid State \& Structural Chemistry Unit, Indian Institute of Science, Bangalore 560012, India.

c. Centre for High Energy Physics, Indian Institute of Science, Bangalore 560012, India.

\begin{abstract}
Generally, the first step in modeling molecular magnets involves obtaining the lowlying eigenstates of a Heisenberg exchange Hamiltonian which conserves total spin and belongs usually to a non-Abelian point group. In quantum chemistry, it has been a long standing problem to target a state which has definite total spin and also belongs to a definite irreducible representation of the point group. Many attempts have been made over years, but unfortunately these have not resulted in methods that are easy to implement, or even applicable to all point groups. Here we present a general technique which is a hybrid method based on Valence Bond basis and the basis of z-component of the total spin, which is applicable to all types of point groups and is easy to implement on computer. We illustrate the power of the method by applying it to the molecular magnetic system, $\mathrm{Cu}_{6} \mathrm{Fe} e_{8}$, with cubic symmetry. We emphasize that our method is applicable to spin clusters with arbitrary site spins and is easily extended to fermionic systems.
\end{abstract}

\section{Introduction}

The field of molecular magnetism has witnessed an explosion in the number of systems that exhibit molecular magnetic phenomena such as quantum resonant tunneling and photomagnetism (see reviews [1, 2]). This explosive growth has also presented challenges to theorists modeling these systems. The problems encountered by theorists begin with modeling the nature of exchange interactions between pairs of magnetic ions. While an electronic many-body Hamiltonian has to be solved for determining the nature of exchange, this is often circumvented by guessing the nature of exchange based on the knowledge of the ligands, electron configuration of the transition metal ion and the geometry of the complex. The second problem concerns with obtaining the eigenstates of the exchange Hamiltonian,

$$
H_{e x}=-\sum_{\langle i j\rangle} J_{i j} \hat{S}_{i} \cdot \hat{S}_{j}
$$

\footnotetext{
${ }^{1}$ shaon@physics.iisc.ernet.in

${ }^{2}$ rajamani@sscu.iisc.ernet.in

3 ramasesh@sscu.iisc.ernet.in

4diptiman@cts.iisc.ernet.in
} 
describing the coupling between pairs of magnetic ions $\langle i j\rangle$ with exchange constant $J_{i j}$. Often, the Fock space of the Hamiltonian of the magnetic system could be very large (in case of $M n_{12} A c$, it is as large as a hundred million [4] and in $F e_{12}$ ferric wheel, it is more than two billion [3]) and obtaining even a few low-lying states of the Hamiltonian could pose a challenge. Since the exchange Hamiltonian conserves both total spin and z-component of total spin $\left(M_{S}\right)$, the problem can be simplified by specializing the basis, in which the matrix representation of the Hamiltonian is sought, to the case of fixed z-component of the total spin. Further simplification could come from exploiting spatial symmetries of the model. An ideal situation would correspond to one in which all the spin and spatial symmetries are utilized to construct a fully symmetrized basis to minimize the size of the Hamiltonian matrix that needs to be diagonalized.

The conservation of the $S_{\text {tot }}^{z}$, the total z-component of spin is trivially achieved by choosing from the Fock space, states whose total $M_{S}$ value corresponds to the desired value. This is possible since individual $S_{i}^{z}$ operators commute with the $S_{\text {tot }}^{z}$ operator. It is also quite straightforward to set up the Hamiltonian matrix in this basis and solve for a few low-lying states in cases where the Hilbert space is spanned by a few hundred million states [30]. However, the exchange Hamiltonian of molecular magnets often consists of interactions that are geometrically frustrated. In such a system, the ground state spin is often not predictable and one needs to obtain the lowest state in each total spin subspace to fix the spin of the ground state. Besides, low energy states with different total spins lie close in energy and it is numerically difficult to achieve convergence to nearly degenerate eigenstates unless they can be dispersed into orthogonal Hilbert spaces. We can partially alleviate this problem by employing the parity symmetry of the exchange Hamiltonian. This symmetry corresponds to rotation of all the spins in the system around the $\mathrm{x}$ or $\mathrm{y}$-axis by an angle $\pi$ which leaves the Hamiltonian invariant, in the $M_{S}=0$ sector.

$$
\left[H, R_{y}(\pi)\right]=0 ; \text { where, } R_{y}(\theta)=e^{-i \theta \hat{S}_{y} / \hbar}
$$

The action of parity operator $(\hat{P})$ on a basis state with site $m_{s}$ values $m_{1}, m_{2}, m_{3} \ldots m_{n}$, is to flip all the spins in the system, i.e.,

$$
\hat{P}\left|m_{1} m_{2} \ldots m_{n}\right\rangle=(-1)^{\eta}\left|-m_{1}-m_{2} \ldots-m_{n}\right\rangle ; \text { where, } \eta=\sum_{i} s_{i}
$$

Thus, the parity operator which conserves the total $M_{S}$ value, only when $M_{S}=0$, is a symmetry element of the Hamiltonian matrix in the $M_{S}=0$ sector. In the general case where the individual objects have spin $s_{i}$, if $\sum_{i} s_{i}$ is even then symmetric (antisymmetric) combination of the basis states, under parity, will span a space of even (odd) total spin states. The method can be extended easily to systems which allow only half odd-integer total spin. Since in most cases, the lowest excited state usually has a spin which is one different from that of the ground state, this symmetry renders it easy to obtain the spin gap accurately. However, the size of the Hilbert space is only reduced by approximately half of the size of the full $M_{S}=0$ space, 
by using this symmetry. Use of parity is still advantageous as exploiting spatial symmetries is straightforward, even when the point group is not Abelian.

Construction of spin adapted configuration state functions (CSF) has been a problem of long standing interest in quantum chemistry. The CSFs are simultaneous eigenstates of total $S_{\text {tot }}^{2}$ and $S_{\text {tot }}^{z}$ and setting up the Hamiltonian matrix in this basis leads to matrices of smaller size besides allowing automatic labeling of the states by the total spin. Besides, the eigenvalue spectrum is enriched, since we can obtain several low-lying states in each total spin sector. This is in contrast to obtaining several low-lying states in a given total $\mathrm{M}_{S}$ sector as the latter would have states with total spin $\mathrm{S}_{t o t} \geq \mathrm{M}_{S}$. There are many ways of achieving this task [5]. The simplest method involves setting up the matrix of the total spin operator, $S_{t o t}^{2}$, in the CSF basis of fixed $M_{S}$ and obtain the eigenstates corresponding to given total spin value; these eigenstates which are linear combinations of the constant $M_{S}$ CSFs are then the spin adapted CSFs. For large systems this method is not practical. Another method which is some times used is the Löwdin projection method [6, 7] in which a projection operator $P_{S}$, given by,

$$
P_{S}=\Pi_{S^{\prime} \neq S}\left(\hat{S}^{2}-S^{\prime}\left(S^{\prime}+1\right)\right)
$$

is used to project out all undesired states of spins $S^{\prime}$ except the spin $\mathrm{S}$ of interest, from a given CSF. The methods that have been extensively in vogue for construction of the spin adapted CSFs are the Graphical Unitary Group Approach (GUGA) [8, 9, 10], Symmetry Group Graphical Approach (SGGA) [11] and the Valence Bond (VB) approach [12, 13, 14, 15, 17]. In the GUGA method, the total spin adapted CSFs are represented as Shavitt graphs or Paldus arrays and the matrix element of a term (which corresponds to a generator of the unitary group) in the Hamiltonian between any two CSFs is obtained by comparing the two arrays or graphs corresponding to the CSFs. Similarly, graphs are used to represent spin adapted CSFs in the SGGA method and rules for computing matrix elements between two CSFs of a term in the Hamiltonian have been derived (see for example reviews [18, 19, 20]). Using these methods it is possible to carry out large scale configuration interaction (CI) calculations. While in all these methods the total spin symmetry is fully exploited, spatial symmetry adaptation is not an easy task [18]. The CSFs are each built up of several orbitals with each orbital in general transforming according to some specific irreducible representation of the point group of the system. The direct product of the irreducible representations of a general symmetry group is not a single irreducible representation of the same group. Thus it is not possible to associate an irreducible representation with a given CSF unless the point group to which the system belongs is an Abelian group [21, 22]. Otherwise, symmetry operation on a CSF leads to a linear combination of many CSFs which is in general difficult to construct. For small dimensionalities of the Hilbert spaces, matrix representation of the symmetry operators can be obtained in the space of CSFs. The projection matrix for a given irreducible representation can be constructed from these matrices and from these, the symmetry adapted CSFs [23]. However, this approach is of limited value in real large scale problems [18]. In quantum chemical literature this difficulty is bypassed by dealing with Abelian subgroups of the systems point group [24, 25]. But, this can lead to ambiguities in assigning the irreducible representation of a state [26]. Among the methods for constructing 
spin adapted CSFs, the VB method is the simplest and will be considered in the next section.

The ultimate goal of symmetry adaptation is to be able to exploit the full spatial and spin symmetries of the system, both for computational efficiency and for labeling of the state by the irreducible representation to which it belongs. In the next section, we give a brief introduction to the symmetrized valence bond (VB) approach that was developed earlier and highlight the difficulties associated with the technique [16]. In the third section, we present a hybrid VBconstant $M_{S}$ method which overcomes these difficulties. In the fourth section, we illustrate an application of this method to a magnetic spin cluster. In the final section, we summarize and discuss the future prospects for the technique.

\section{The Symmetrized VB Approach}

Exploiting the invariance of both total spin and its z-component is nontrivial, since eigenstates of the $S_{\text {tot }}^{z}$ operator expressed as a product of the eigenstates of all the $S_{i}^{z}$ operators are not simultaneously eigenstates of the $S_{\text {tot }}^{2}$ operator. The situation is further complicated by the fact that in a molecular magnet, often the spins of all the constituent magnetic centers, $s_{i}$ are not the same. In such a situation, the easiest way of constructing the spin adapted functions is the diagrammatic valence bond (VB) method based on modified Rumer-Pauling rules [12, 14]. In this method, a magnetic site with a given spin " $s_{i}$ " is replaced by $2 s_{i}$ spin-half objects. To obtain a state with total spin $S$ from N such spin-1/2 objects from all the magnetic centers, $(N-2 S)$ of these spin-1/2 objects are singlet spin paired explicitly, subject to the following restrictions: (1) there should be no singlet pairing of any two spin-half objects belonging to the same magnetic center (this ensures that the $2 s_{i}$ objects are in a totally symmetric combination [29]), (2) a total of $2 S$ spin-half objects are left unpaired, (3) when all the spin-half objects are arranged at the vertices of a regular polygon with number of vertices equal to number of spin-half objects, $\mathrm{N}$, and straight lines are drawn between spin paired vertices, there should be no intersecting lines in the resulting diagram and (4) when all the spin-half objects are arranged on a straight line and lines are drawn between spin paired objects, these lines should not enclose any unpaired spin$1 / 2$ object. These rules follow from the generalization of the Rumer-Pauling rules to objects with spin greater than $1 / 2$ and total spin greater than zero. The set of diagrams which obey these rules would hence forth be called "legal" VB diagrams. Some legal VB diagrams are shown in Fig. 1. A line in the VB diagram between two spin $-1 / 2$ objects $i$ and $j$ corresponds to the state $\left(\alpha_{i} \beta_{j}-\beta_{i} \alpha_{j}\right) / \sqrt{2}$ where we choose $\alpha$ to correspond to $|\uparrow\rangle$ and $\beta$ to $|\downarrow\rangle$ orientations of the spin. The phase convention assumed is that the ordinal number " $i$ " is less than the ordinal number " $j$ ". The $2 S$ spin-1/2 objects $k_{1} k_{2} k_{3} \ldots k_{2 S}$ which are left unpaired can be taken to represent the state with $M_{S}=S$ given by $\alpha_{k_{1}} \alpha_{k_{2}} \alpha_{k_{3}} \ldots \alpha_{k_{2 s}}$. VB states corresponding to other $\mathrm{M}_{S}$ value for this state with spin $\mathrm{S}$, can be obtained by operating the $\mathrm{S}_{\text {tot }}^{-}$operator on the state by the required number of times. Since the exchange Hamiltonian is isotropic, each eigenstate in the spin $\mathrm{S}$ is $(2 \mathrm{~S}+1)$ fold degenerate and it is sufficient to work in subspace of chosen $\mathrm{M}_{S}$ value. The VB state corresponding to a given diagram is a product of the states representing the constituent parts of the diagram. On a computer, a "legal" VB diagram of any spin can be 


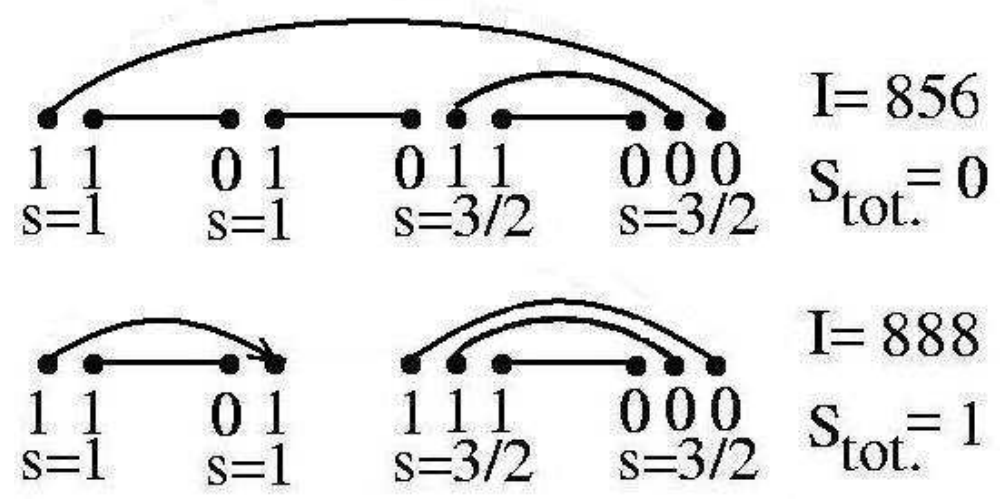

Figure 1: Above VB diagram shows spin pairings to yield a total spin $S_{t o t}=0$ state from ten spin-1/2 objects, constituent elementary spins of two spin 1 and two spin $3 / 2$. Its bit representation corresponds to unique integer $\mathrm{I}=856$. The bottom VB diagram shows a $S_{\text {tot }}=1$ state, the corresponding unique integer is $\mathrm{I}=888$.

uniquely represented by an integer of $2 \mathrm{~N}$ bits with a one bit representing the beginning of a singlet line and a zero bit the ending of singlet line. The unpaired spins are also represented as one-bits. Fig. 1 also shows bit representation of typical VB diagrams.

To spatially symmetrize a VB basis, it is necessary to know the result of a symmetry operator operating on a legal VB diagram. In general, the resultant of such an operation on a "legal" VB diagram is an "illegal" VB diagram. An example of this is shown in Fig. 2 [16]. An illegal VB diagram can be decomposed into a linear combination of legal VB diagrams, a process that is computationally demanding. In practice, the VB space is broken down into smaller invariant subspaces of the symmetry group and within each invariant space, a symmetrized linear combination of the VB basis is constructed. However, the structure of the invariant spaces is very complex and constructing disjoint invariant spaces is not simple. While constructing symmetrized VB basis, a projection operator for a given symmetry representation is employed to project the symmetrized linear combinations by acting on each of the VB states in the invariant space. While the number of linearly independent symmetry combinations of a given representation is known a priori, the actual linear combinations are obtained by carrying out Gram-Schmidt orthonormalization of the projected states. However, since the VB diagrams are not orthogonal the orthonormalization process is both computationally involved and time consuming.

Furthermore, in case of molecular magnets containing magnetic ions with spin greater than half, the exchange operator between such high-spin centers also generates "illegal" VB diagrams as it involves non-nearest neighbor exchange interactions between constituent elementary spins [14]. To illustrate, exchange between a center $\mathrm{A}$ with say spin one and a center $\mathrm{B}$ with spin $3 / 2, S_{A} \cdot S_{B}$, is expressed as $\left(s_{A_{1}}+s_{A_{2}}\right) \cdot\left(s_{B_{1}}+s_{B_{2}}+s_{B_{3}}\right)$. These exchange terms operate on a VB diagram with constituent elementary spins which are non-nearest neighbors and 



Figure 2: The effect of operation by the $C_{3}^{1}$ symmetry operator about the $(1,8)$ axis. Top left shows the initial and final VB diagrams with spin couplings between vertices of the cube shown as dark lines. Bottom left shows the same states as spin couplings between vertices of a regular octagon. The resultant is an "illegal" diagram. Decomposing the resultant to "legal" VB diagrams yields a sum of five VB diagrams shown on the right, with spin couplings between vertices on a cube.

in general generate "illegal" VB diagrams as per the VB rules. Decomposition of the resultant "illegal" VB diagrams to "legal" VB diagrams could present a serious bottle-neck in computing the Hamiltonian matrix elements. In view of these difficulties, a fully symmetrized VB approach to solving Heisenberg exchange Hamiltonian particularly in the context of molecular magnets is not feasible.

\section{Hybrid method based on VB Basis and Constant $M_{S}$ Basis}

In the constant $M_{S}$ basis, a basis state of an ensemble of spins $s_{1}, s_{2}, \cdots, s_{N}$, is represented by a direct product of the $m_{s}$ states of each spin such that the total $M_{S}=\sum m_{i}$. The basis states in the constant $M_{S}$ representation are orthonormal by construction. Given the definition of a line in the VB diagram, every VB diagram can be broken up into a linear combination of the constant $M_{S}$ basis states. A VB diagram with $p$ singlet lines give rise to $2^{p}$ basis states in the constant $M_{S}$ basis. To effect the conversion of VB diagrams to constant $M_{S}$ functions, each singlet line gives two states; in one state, the site at which a singlet line begins is replaced by an $\alpha$ spin and the one at which it ends by a $\beta$ spin with phase +1 and in the other the spins are reversed and the associated phase is -1 . Once the VB diagrams are converted to constant $M_{S}$ basis states with constituent spins, it is possible to associate a $m_{i}$ value with a composite spin, given by $m_{i}=\left(n_{i \uparrow}-n_{i \downarrow}\right) / 2$, where $n_{i \uparrow}$ is the number of up-spin halves and $n_{i \downarrow}$ is the number of down-spin halves at the site $\mathrm{i}$. However, there is a normalization constant $\mathrm{w}_{i}$, which follows from Clebsch-Gordan coefficients, given by,

$$
w_{i}=\left[\frac{\left(2 s_{i}\right) !}{\left(s_{i}+m_{i}\right) !\left(s_{i}-m_{i}\right) !}\right]^{-1 / 2}
$$


for a site with spin $s_{i}$ in state $m_{i}$ [29]. We can assume without loss of generality that the $M_{S}$ value of the VB diagram is also $S$. Computationally, finding the transformation of a state in the VB basis to constant $M_{S}$ basis is straightforward. We initialize the coefficients in the constant $M_{S}$ basis to zero. We then decompose, sequentially, each VB diagram into constant $M_{S}$ states and update the coefficient of the basis state of corresponding $M_{S}$ by adding to it the VB coefficient times the product of Clebsch-Gordan factors with appropriate phases. The matrix relating the VB basis states to constant $M_{S}$ basis states, $\mathbf{C}$, is a $V \times M$ matrix, where $V$ is the dimensionality of the VB space and $M$ that of the constant $M_{S}$ space.

The construction of the projection operator for projecting all the states of a given symmetry representation in a given spin space can now be accomplished. We construct the matrix representation of a symmetry operator, $\hat{R}$, of the point group in the chosen spin space by operating with $\hat{R}$ on each state in the constant $M_{S}$ basis and searching for the resulting state in the list of $M_{S}$ basis states. Each basis state in this representation is carried over to another basis state by a symmetry operation of the point group. Thus, the matrix $\mathbf{R}_{M}$ though an $M \times M$ matrix contains only one nonzero element in each row; this makes manipulations with this matrix computationally fast. The knowledge of the $\mathbf{C}$ and the $\mathbf{R}_{M}$ matrices give the effect of operating by the symmetry operator $\hat{R}$ on a VB state as a linear combination of the constant $M_{S}$ basis states via the matrix $\mathbf{B}_{R}=\mathbf{C R}_{M}$. The projection operator for projecting out the basis states on to a chosen irreducible representation of the point group $\Gamma$ is given by,

$$
P_{\Gamma}=\sum_{\hat{R}} \chi_{\Gamma}^{i r r}(\hat{R}) \hat{R}
$$

where, $\chi_{\Gamma}^{i r r}(\hat{R})$ is the character under the symmetry operation $\hat{R}$ in the character table of the point group of the system [31]. The matrix representation of $P_{\Gamma}$ in the mixed VB and constant $M_{S}$ basis is given by,

$$
\mathbf{Q}_{\Gamma}=\sum_{R} \chi_{\Gamma}^{i r r}(R) \mathbf{B}_{R}
$$

where, $\mathbf{Q}_{\Gamma}$ is a $V \times M$ matrix. However, the rows of the matrix $\mathbf{Q}_{\Gamma}$ are not linearly independent, since the symmetrized basis spans a much smaller dimensional Hilbert space. The exact dimension of the Hilbert space spanned by the system in the irreducible representation $\Gamma$ can be known a priori. The dimension of the space $\Gamma, \mathrm{V}_{\Gamma}$, is given by,

$$
V_{\Gamma}=\left(d_{\Gamma} / h\right) \sum_{\hat{R}} \chi(\hat{R}) \chi_{\Gamma}^{i r r}(\hat{R})
$$

where $d_{\Gamma}$ is the dimensionality of the irreducible representation $\Gamma, h$ is the number of elements in the point group and $\chi(\hat{R})$ is the reducible character for the operation $\hat{R}$. The $V_{\Gamma} \times M$ projection matrix, $\mathbf{P}_{\Gamma}$ of rank $V_{\Gamma}$ is obtained by Gram-Schmidt orthonormalization of the rows of the matrix 
$\mathbf{Q}_{\Gamma}$ until $V_{\Gamma}$ orthonormal rows are obtained. These $V_{\Gamma}$ orthonormal rows represent the projection matrix $\mathbf{P}_{\Gamma}$. The $M \times M$ Hamiltonian matrix, $\mathbf{H}_{M}$ is constructed in the constant $M_{S}$ basis which is described elsewhere [30]. Since the basis states in this representation are orthonormal, we do not encounter the problem of "illegal" states as with the VB representation. The $V_{\Gamma} \times V_{\Gamma}$ Hamiltonian matrix in the fully symmetrized basis is given by $\mathbf{P}_{\Gamma} \mathbf{H}_{M}\left(\mathbf{P}_{\Gamma}\right)^{\dagger}$ and one could use any of the well known full diagonalization routines to obtain the full eigenspectrum or use Davidson algorithm to get a few low-lying states of the symmetrized block Hamiltonian in the chosen spin and symmetry subspace.

The above procedure does not lead to the smallest block of the Hamiltonian matrix, when the irreducible representation for the block is degenerate, such as the E, $\mathrm{T}$ or $\mathrm{H}$ representations. In such cases, it is advantageous to work with bases that transform according to one of the components of the irreducible representation. This can be achieved by choosing an axis of quantization and projecting out bases states of the irreducible representation which are diagonal about a rotation about the quantization axes. For example, in the case of the irreducible representation that transforms as $\mathrm{T}$, we can choose one of the $\mathrm{C}_{3}$ axes as a quantization axis and project the basis states of the irreducible representation using $\left(I+C_{3}^{1}+C_{3}^{-1}\right)$ as the projection operator. This operator projects states that transform as the $\mathrm{Y}_{1}^{0}$ component of the three fold degenerate irreducible tensor operator. Similarly, for the E representation, we could use any of the $\mathrm{C}_{2}$ axis as a quantization axis and use the projection operator $\left(\mathrm{I}+\mathrm{C}_{2}^{1}\right)$ to project out basis states that transform as one of its components. This is equivalent to projecting out the states which transform as a given row of the irreducible representation; the latter are not listed in standard group theoretical character tables.

Computation of static properties such as spin densities and spin-spin correlation functions in the eigenstates of the Hamiltonian is rendered simple due to the orthogonality of the constant $M_{S}$ basis. The site spin operators such as the z-component of the spin are diagonal in this basis, while other operators such as the raising and lowering operators, though not diagonal in this basis, have a very simple matrix representation. In computing various properties, the procedure we follow is to express the eigenstates in the unsymmetrized constant $M_{S}$ basis and to compute the desired properties using this representation.

The additional steps involved in the hybrid VB-Constant $\mathrm{M}_{S}$ method are (i) constructing the $\mathrm{C}$ matrix, whose $\mathrm{i}^{\text {th }}$ row contains the coefficients of the constant $\mathrm{M}_{S}$ functions appearing in the $\mathrm{i}^{\text {th }}$ VB basis function. This is a very fast step as the constant $\mathrm{M}_{S}$ states are an ordered sequence of integers and a VB state with $n$ lines is a linear combination of $2^{n}$ constant $\mathrm{M}_{S}$ functions. (ii) In the hybrid approach, computation of the $\mathbf{C}$ matrix involves the matrix multiplication, $\mathbf{C}\left(\sum_{R} \chi_{\Gamma}^{i r r}(R) \mathbf{R}_{M}\right)=\mathbf{C} \mathbf{R}_{M}^{\prime}$. The number of arithmetic operations involved is however very small, since both $\mathbf{C}$ and $\mathbf{R}_{M}^{\prime}$ are sparse matrices. In both constant $\mathbf{M}_{S}$ and hybrid approaches one has to obtain the projection operator $\mathbf{P}_{\Gamma}$ by retaining only the orthogonal rows of the matrix $\mathbf{R}_{M}^{\prime}$ or $\mathbf{Q}_{\Gamma}$ respectively. Since the number of orthogonal rows in $\mathbf{Q}_{\Gamma}$ is far fewer than in $\mathbf{R}_{M}^{\prime}$, this step is faster in the hybrid approach than in the constant $M_{S}$ approach by a factor $\mathrm{D}\left(\Gamma_{S}\right) / \mathrm{D}\left(\Gamma_{M_{S}}\right)$, where $\mathrm{D}\left(\Gamma_{S}\right)$ is the dimensionality of the space of the irreducible representation $\Gamma$ with spin $\mathrm{S}$ and $\mathrm{D}\left(\Gamma_{M_{S}}\right)$ is similarly the dimension of the space $\Gamma$ with constant $\mathrm{M}_{S}$. This advantage is largely off-set by the fact that the $\mathbf{R}_{M}^{\prime}$ matrix in constant $M_{S}$ basis is more sparse than the $\mathbf{Q}_{\Gamma}$ 


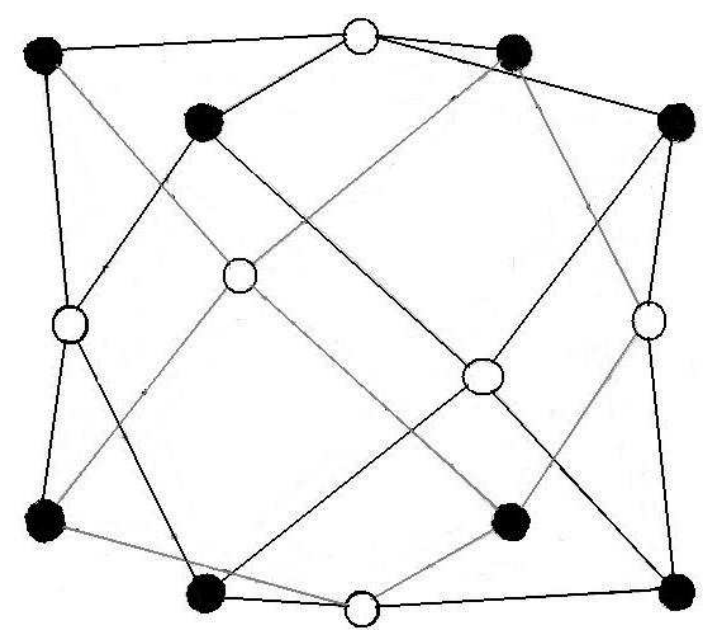

Figure 3: Schematic of $\mathrm{Cu}_{6} \mathrm{Fe}_{8}$ cluster. Filled and open circles correspond to $\mathrm{Fe}$ and $\mathrm{Cu}$ (both spin-1/2) sites respectively. Lines represent the exchange coupling between various spin sites.

matrix in the hybrid approach.Computation of the eigenvalues in the constant $M_{S}$ approach is slower than in the hybrid approach, since $\mathrm{D}\left(\Gamma_{M_{S}}\right)>\mathrm{D}\left(\Gamma_{S}\right)$ for most $\mathrm{S}$. This advantage may be partially off-set because the Hamiltonian matrix in the hybrid approach is usually more dense. The memory required for the hybrid approach is not very different from that of constant $M_{S}$ approach since the matrices though smaller in the hybrid approach, are slightly denser. The only additional array required in the hybrid approach is the storage of $\mathbf{C}$ matrix, which is very sparse. The major advantage of the hybrid approach is that we can obtain a far richer spectrum, since we are targeting each spin sector separately, unlike in the constant $M_{S}$ approach. Thus, if we can obtain, say 10 states in each $S$ sector of the $2 n$ spin- $1 / 2$ problem, we would have $10(n+1)$ unique states compared to the constant $M_{S}$ technique where many of these spin states would be repeated in different $M_{S}$ sectors.

\section{Application to $\mathrm{Cu}_{6} \mathrm{Fe}_{8}$}

We have applied the above method to model the susceptibility behavior of the molecule $\left[(T p)_{8}\right.$ $\left.\left(\mathrm{H}_{2} \mathrm{O}\right)_{6} \mathrm{Cu}_{6}^{\mathrm{II}} \mathrm{Fe}_{8}^{\mathrm{III}}(\mathrm{CN})_{24}\right]\left(\mathrm{ClO}_{4}\right)_{4} \cdot 12 \mathrm{H}_{2} \mathrm{O} \cdot 2 \mathrm{Et}_{2} \mathrm{O}$ [27], where Tp stands for hydrotris (pyrazolyl) borate (Fig. 3). In this molecule the $C u^{I I}$ ions as well as the $F e^{I I I}$ are in spin-1/2 state. The eight $F e^{I I I}$ ions are at cube corners and the six $C u^{I I}$ ions are on the outward perpendicular to the face centers of the cube. Each $C u^{I I}$ ion is connected to the four nearest $F e^{I I I}$ ions via ferromagnetic exchange interactions. There are no $\mathrm{Fe}-\mathrm{Fe}$ or $\mathrm{Cu}-\mathrm{Cu}$ interactions. The molecule has a spin 7 ground state. This system has a very high symmetry of the cube and incorporates all the complexities that can be encountered in the application of our technique. From the susceptibility data, the strength of the exchange interaction $\mathrm{J}$, was estimated to be $30 \mathrm{~cm}^{-1}$ [27]. Because of the high symmetry of this system, we chose this as an example for applying our 
Table 1: Dimensionalities of the total spin spaces of a system of 14 spin-1/2 objects. $D(S)$ is the dimensionality of the constant $\mathrm{S}$ basis and $D\left(M_{S}\right)$ is the dimensionality of the constant $M_{S}$ basis.

\begin{tabular}{|l||c|c|c|c|c|c|c|c|}
\hline $\mathrm{S} / \mathrm{M}_{S}$ & 0 & 1 & 2 & 3 & 4 & 5 & 6 & 7 \\
\hline$D(S)$ & 429 & 1001 & 1001 & 637 & 273 & 77 & 13 & 1 \\
\hline$D\left(M_{S}\right)$ & 3432 & 3003 & 2002 & 1001 & 364 & 91 & 14 & 1 \\
\hline
\end{tabular}

Table 2: Dimensionalities of various subspaces of the $C u_{6}^{I I} F e_{8}^{I I I}$ cluster for irreducible representation $\Gamma$.

\begin{tabular}{|c||c|c|c|c|c|c|c|c|}
\hline $\begin{array}{c}\mathrm{S}_{\text {tot }} \rightarrow \\
\Gamma \downarrow\end{array}$ & 0 & 1 & 2 & 3 & 4 & 5 & 6 & 7 \\
\hline \hline $\mathrm{A}_{1 g}$ & 6 & 32 & 24 & 24 & 9 & 5 & 1 & 1 \\
\hline $\mathrm{A}_{2 g}$ & 13 & 15 & 19 & 8 & 5 & 0 & 0 & 0 \\
\hline $\mathrm{E}_{g}$ & 34 & 90 & 90 & 60 & 26 & 10 & 2 & 0 \\
\hline $\mathrm{T}_{1 g}$ & 78 & 165 & 171 & 99 & 39 & 6 & 0 & 0 \\
\hline $\mathrm{T}_{2 g}$ & 66 & 216 & 186 & 138 & 54 & 21 & 3 & 0 \\
\hline $\mathrm{A}_{1 u}$ & 5 & 19 & 13 & 11 & 2 & 0 & 0 & 0 \\
\hline $\mathrm{A}_{2 u}$ & 17 & 20 & 27 & 15 & 10 & 2 & 1 & 0 \\
\hline $\mathrm{E}_{u}$ & 36 & 78 & 84 & 48 & 20 & 6 & 0 & 0 \\
\hline $\mathrm{T}_{1 u}$ & 105 & 180 & 219 & 123 & 66 & 15 & 6 & 0 \\
\hline $\mathrm{T}_{2 u}$ & 69 & 186 & 168 & 111 & 42 & 12 & 0 & 0 \\
\hline
\end{tabular}

technique. The dimensions of the various subspaces are given in Table 1.

Using the hybrid VB-constant $M_{S}$ method, we have broken down the space in each total spin sector into basis states that transform as different irreducible representations of the cubic point group. The dimensionalities of the various subspaces are shown in Table 2. The subspaces transforming as the E representations are broken down into subspaces of half their dimension by quantizing the system along a $\mathrm{C}_{2}$ axis. Similarly, the subspaces transforming as the $\mathrm{T}$ representations are broken down into one third their dimensions in Table 2, by using a $\mathrm{C}_{3}$ axis as the axis of quantization.

We have set up the Hamiltonian matrix in each of the subspaces and obtained all the eigenvalues. We have also used a constant $M_{S}$ basis and using the full cubic symmetry, factored the space into various irreducible representations and obtained all the eigenvalues in each subspace. From the eigenvectors, we have computed the total spin of the state. We find a one to one correspondence to numerical accuracy, between the two sets of calculations. We have also fitted the $\chi T$ vs. T experimental plot by using the full spectrum of the Heisenberg Hamiltonian and computing [28] 


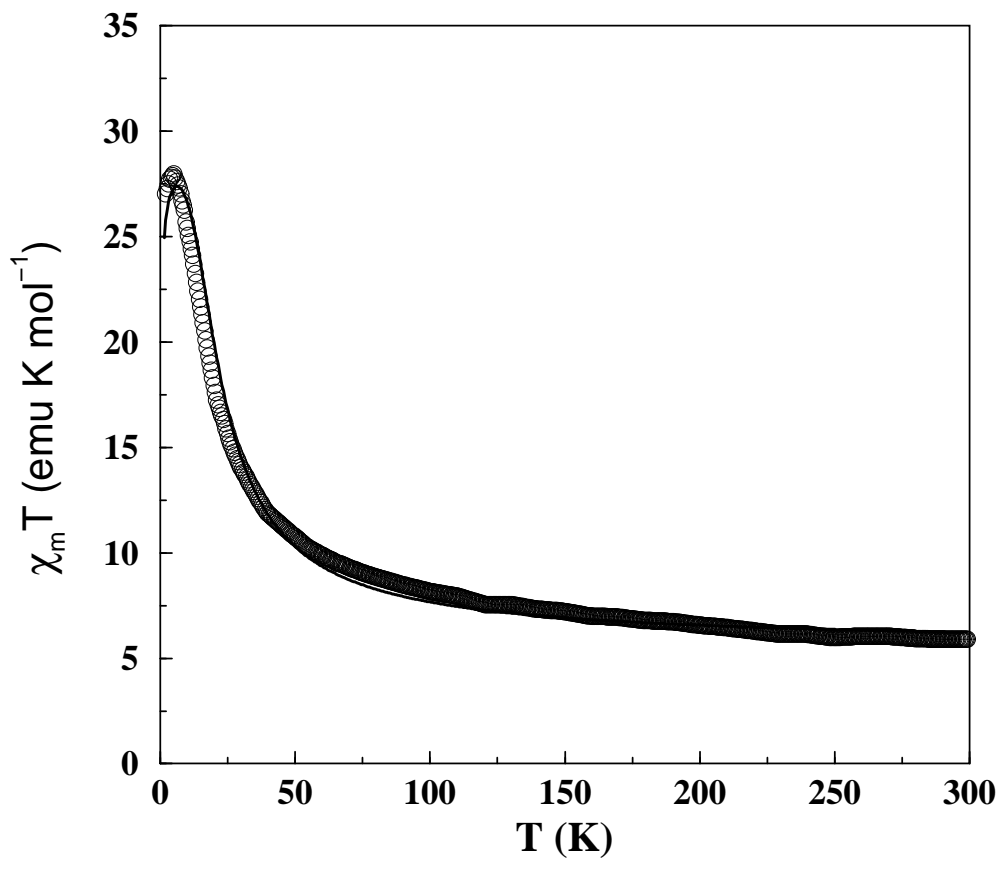

Figure 4: Fit of the $\chi T$ vs. T plot for the $C u_{6}^{I I} F e_{8}^{I I I}$ cluster. The best fit parameters are, $\mathrm{J}=27.2$ $\mathrm{cm}^{-1}$ (ferromagnetic), $z J^{\prime}=-0.008 \mathrm{~cm}^{-1}$ (antiferromagnetic), $\mathrm{D}=-0.15 \mathrm{~cm}^{-1}$ and $\mathrm{g}=2.1$.

$$
\chi T=\frac{3}{8}\left[\frac{g^{2} F(J, T)}{1-z J^{\prime} F(J, T) / k_{B} T}\right]
$$

where, we have taken the $\mathrm{g}$ factor to be 2.1, the ferromagnetic exchange constant $\mathrm{J}$ to be 27.2 $\mathrm{cm}^{-1}$. Here, $\chi T$ is in units of $N \mu_{B}$. The function $\mathrm{F}(\mathrm{J}, \mathrm{T})$ is given by,

$$
F(J, T)=\frac{\sum_{S} \sum_{M_{S}} M_{S}^{2} \exp \left[-E_{0}\left(S, M_{S}\right) / k_{B} T\right]}{\sum_{S} \sum_{M_{S}} \exp \left[-E_{0}\left(S, M_{S}\right) / k_{B} T\right]}
$$

with $E_{0}\left(S, M_{S}\right)$ being the eigenvalue of the sum of exchange Hamiltonian and the magnetic anisotropy term $D S_{Z}^{2}$ and $z J^{\prime}$ is the intermolecular exchange interaction. Here we have assumed that the molecular anisotropy is along the global z-axis, and this term is treated as a perturbation to the exchange Hamiltonian in Eq. 1. In Fig. 4, we show the fit of the experimental data to the model. 


\section{Summary and Outlook}

The problem of exploiting total spin invariance together with spatial symmetries, especially of non-Abelian point groups has been a long standing one. While full spin symmetry adaptation can be achieved by various techniques such as the use of permutation groups, unitary groups and the VB method, the last mentioned is the easiest and provides easy chemical visualization of the basis states. The main objection to the VB technique had been that the basis is nonorthogonal and leads to nonsymmetric representation of the Hamiltonian matrix. However, with the modification of the Davidson's algorithm [32] to nonsymmetric matrices by Rettrup [33], this objection has ceased to be important. The ease with which VB states with any given total spin can be generated from objects with assorted individual spins, is an advantage which far outweighs the other historical objections to the VB method.

However, even the VB basis suffers from the serious disadvantage like all other spin adapted methods, when the question of full spatial symmetry adaptation comes up. The constant $M_{S}$ basis methods do not suffer from this disadvantage. In this paper, we have demonstrated, how by combining the ease of spin symmetry adaptation of the VB method with the spatial symmetry exploitation of the constant $M_{S}$ methods, we can devise a scheme which is fully spin and spatial symmetry adapted. This has been possible because of the ease of transformation of the VB basis to the constant $M_{S}$ basis. We have demonstrated the power of the method by applying it to the exchange Hamiltonian of the molecular magnet $\mathrm{Cu}_{6} \mathrm{Fe}_{8}$ which has cubic symmetry. We note that the hybrid VB-constant $M_{S}$ method also allows easy manipulation of the eigenstates of the Hamiltonian for computing properties. The method described here can easily be extended to fermionic systems and should provide a significant improvement for obtaining exact eigenstates of spin conserving model Hamiltonians.

Acknowledgement This work was supported through project No. 3108-3 funded by the IndoFrench Centre for Promotion of Advanced Research (IFCPAR) and by the Swedish Research Council through their grant No. 348-2006-6666.

\section{References}

[1] D. Gatteschi and R. Sessoli, Angew. Chem. Int. Ed. 42, 268 (2003).

[2] D. Gatteschi and R. Sessoli, J. Magn. Magn. Mater. 272-276, 1030 (2004).

[3] I. Rudra, S. Ramasesha and D. Sen, Phys. Rev. B 66, 014441 (2002).

[4] C. Raghu, I. Rudra, D. Sen and S. Ramasesha, Phys. Rev. B 64, 064419 (2001).

[5] R. Pauncz, Spin Eigenfunctions: Construction and Use, Plenum Press: New York, 1979.

[6] P.-O. Löwdin, Phys. Rev. 97, 1509 (1955).

[7] B. Bernu, P. Lecheminant, C. Lhuillier and L. Pierre, Phys. Rev. B 50, 10048 (1994). 
[8] P. Saxe, D. J. Fox, H. F. Schaefer and N. C. Handy, J. Chem. Phys. 77, 5584 (1982).

[9] P. E. M. Siegbahn, J. Chem. Phys. 72, 1647 (1980).

[10] B. R. Brooks and H. F. Schaefer, J. Chem. Phys. 70, 5092 (1979).

[11] W. Duch and J. Karwowski, Int. J. Quantum Chem. 22, 783 (1982).

[12] L. Pauling, J. Chem. Phys. 1, 280 (1933); L. Pauling and G. W. Wheland, ibid, 1, 362 (1933).

[13] H. Eyring, J. Walter and G. E. Kimball, Quantum Chemistry, Wiley: New York, 1944, chap. 13.

[14] Z. G. Soos and S. Ramasesha, in Valence Bond Theory and Chemical Structure, D. J. Klein, N. Trinajstic, Ed.; Elsevier: New York, 1990, p. 81; S. Ramasesha, and Z. G. Soos, in Valence Bond Theory, D. L. Cooper, Ed.; Elsevier: 2002, chap. 20.

[15] B. Sinha, I. D. L. Albert and S. Ramasesha, Phys. Rev. B 42, 9088 (1990).

[16] S. Ramasesha and Z. G. Soos, J. Chem. Phys. 98, 4015 (1993).

[17] S. Mazumdar and Z. G. Soos, Synth. Metals 1, 77 (1979).

[18] W. Duch, J. Mol. Struc. (Theochem) 234, 27 (1991).

[19] W. Duch and J. Karwowski, Comput. Phys. Rep. 2, 93 (1985).

[20] M. A. Robb and U. Niazi, Comput. Phys. Rep. 1, 127 (1984).

[21] I. Shavitt, Mol. Phys. 94, 3 (1998).

[22] I. Shavitt, in Methods of Electronic Structure Theory, H. F. Schaefer III, Ed.; Plenum Press: New York, 1977, p. 189.

[23] R. J. Buenker and S. D. Peyerimhoff, Theor. Chim. Acta 12, 183 (1968).

[24] S. Rettrup and C. R. Sarma, Theor. Chim. Acta 46, 73 (1977).

[25] I. Shavitt, Chem. Phys. Lett. 63, 421 (1979).

[26] S. Rettrup, C. R. Sarma and J. P. Dahl, Int. J. Quantum Chem. 22, 127 (1982).

[27] Shi Wang et al., Angew. Chem. Int. Ed. 43, 5940 (2004).

[28] O. Kahn, Molecular Magnetism, VSH: Weinheim, 1993.

[29] D. P. Arovas, A. Auerbach and F. D. M. Haldane, Phys. Rev. Letts. 60, 531 (1988).

[30] S. K. Pati, S. Ramasesha, and D. Sen, in Magnetism: Molecules to Materials IV, J. S. Miller and M. Drillon, Ed.; Wiley-VCH: 2002; chap. 4. 
[31] D. M. Bishop, Group Theory and Chemistry, Clarendon Press: Oxford, 1973.

[32] E. R. Davidson, J. Comput. Phys. 17, 87 (1975).

[33] S. Rettrup, J. Comput. Phys. 45, 100 (1982). 